\title{
Characterization of Hemangioblastomas of Spinal Nerves
}

Sven Gläsker, M.D.

Department of Neurosurgery, Albert-Ludwigs-University, Freiburg, Germany

Ansgar Berlis, M.D.

Department of Neuroradiology, Albert-Ludwigs-University, Freiburg, Germany

Axel Pagenstecher, M.D. Department of Neuropathology, Albert-Ludwigs-University, Freiburg, Germany

\section{Vassilios I. Vougioukas,} M.D.

Department of Neurosurgery, Albert-Ludwigs-University, Freiburg, Germany

Vera Van Velthoven, M.D.

Department of Neurosurgery, Albert-Ludwigs-University, Freiburg, Germany

Reprint requests:

Vera van Velthoven, M.D. Neurosurgical University Clinic, Breisacherstraße 64,

D-79106 Freiburg, Germany. Email:

velthov@nz.ukl.uni-freiburg.de
OBJECTIVE: Hemangioblastoma is classified as a benign tumor of the central nervous system. Peripheral nervous system hemangioblastomas to date have been described only in a few case reports. Experience in treating patients with these rare lesions, which harbor diagnostic and therapeutic pitfalls, is limited.

METHODS: To characterize these lesions better, we reviewed our hemangioblastoma database for patients who underwent surgery for extradural hemangioblastoma of the spinal nerve.

RESULTS: Between 1983 and 2003, six patients underwent surgery for spinal nerve hemangioblastomas at our institution. These tumors occurred in $2 \%$ of all patients with hemangioblastomas of the central nervous system, or $6 \%$ of all patients with spinal hemangioblastomas. The occurrence did not differ in von Hippel-Lindau disease cases versus sporadic cases. Radiographically, the tumors easily could be mistaken for schwannomas or metastases; however, they did have some typical features. If a hemangioblastoma was not suspected primarily, profuse bleeding could complicate surgery. Most of the tumors arose from the dorsal sensory fascicles. The vascular supply was from extradural circulation. In general, the surgical outcome of these lesions was good, and permanent neurological deficit was rare. However, local recurrence was observed in three of six patients.

CONCLUSION: These tumors harbor diagnostic and therapeutic pitfalls. In general, the tumors are surgically more challenging, and clinically significant bleeding as well as local tumor recurrence is more common than in intradural hemangioblastomas, mostly because of the frequency of incorrect initial radiographic diagnosis. We suggest that because of the surgical consequences, hemangioblastoma should always be considered to be an important radiological differential diagnosis for nerve sheath tumors. Angiography can bring clarification to ambiguous cases.
C apillary hemangioblastoma is a highly vascular tumor that comprises approximately $3 \%$ of all tumors of the central nervous system (CNS) $(3,10,12,17)$. These tumors occur predominantly in the cerebellum and spinal cord but can be found throughout the CNS. The tumor occurs as a sporadic entity, or in approximately 20 to $30 \%$ as a component tumor of von Hippel-Lindau disease (VHL), an autosomal-dominant inherited disorder with incomplete penetrance and expression (18). The World Health Organization classifies hemangioblastoma as a benign tumor limited to the CNS (1). Hemangioblastoma of the peripheral nervous system so far has not been well described. Only 10 cases of peripheral hemangioblastomas have been reported. Two were case reports of hemangioblastomas of a peripheral nerve of the extremities $(2,9)$, and eight were cases of spinal nerve hemangioblastomas $(4-6,11,16,20)$.

To date, there have been no published series that describe the epidemiology, radiological features, and surgical management of spinal nerve hemangioblastomas. Because these tumors harbor diagnostic and therapeutic pitfalls, we believe that these tumors deserve special attention.

Our institution serves as a referral center for patients with VHL. To characterize these le- 
sions better, we reviewed our large series of hemangioblastoma patients for hemangioblastomas of spinal nerves.

\section{PATIENTS AND METHODS}

\section{Patient Selection Criteria}

Our clinical database includes a large series of patients who underwent surgery for hemangioblastomas. We retrospectively reviewed the data of all patients with the diagnosis of hemangioblastoma treated between 1983 and 2003. Only patients who exhibited an entirely extradural location of the tumor during surgery were included in the study.

\section{Data Evaluation}

Clinical data about the symptoms and neurological outcome of the patients were reviewed. A neuroradiologist (AB) reviewed the magnetic resonance imaging (MRI) scans of the patients for the appearance of the lesions, typical features, and possible differential diagnoses. Operative protocols were reviewed regarding the character and pathological anatomy of these lesions. A neuropathologist (AP) reviewed the specimens of the patients. Special attention was paid to possible differences between these tumors and their CNS counterparts.

\section{Histopathological Examination}

The surgical specimens were embedded in paraffin, and 5 - $\mu$ m-thick sections were prepared. Routine stains included hematoxylin and eosin and reticulin fiber impregnation (Tibor-Pap procedure). Immunohistochemistry analysis was performed with antibodies against S100 protein, neural cell adhesion molecule, and neuron-specific enolase, as described elsewhere (8).

\section{Technique and Methods in Imaging}

In all patients with sporadic tumors, primary and follow-up examinations were performed on a 1.5-T high-field MRI scanner (Siemens Vision; Siemens Medical Systems, Erlangen, Germany). First, T1-weighted images were acquired without contrast. Directly after intravenous contrast administration, axial and sagittal T1-weighted images of the brain were acquired, followed by sagittal T1-weighted images of the complete spine. Before surgery, the regions of interest were examined with axial and sagittal studies with native T1-weighted images, T1-weighted images with contrast, and T2-weighted images. In all patients with VHL, only contrast-enhanced images were acquired. In general, spinal angiography was performed in two circumstances: if there was uncertainty about the diagnosis or if we considered the spinal arterial supply to be at significant risk.

\section{RESULTS}

\section{Epidemiological Characteristics}

A total of 277 patients have been treated for hemangioblastomas of the CNS in our institution between 1983 and 2003, many of them several times. Of these patients, 58\% had VHL. These patients are certainly overrepresented in our center because of its role as a VHL referral center. On an epidemiological basis, as we have calculated, approximately $22 \%$ of all hemangioblastoma patients have VHL (10).

Of all hemangioblastoma patients, $38 \%$ had spinal tumors ( $17 \%$ had posterior fossa tumors additionally). Spinal nerve hemangioblastoma was observed in six patients. Because only symptomatic tumors or tumors that exhibited progression on serial MRI scans were operated on, asymptomatic tumors were not included in this study, which only includes surgical patients. Thus, we can give only an approximation of the incidence of these tumors. Hemangioblastoma of the spinal nerve occurs in approximately $2 \%$ of all patients with CNS hemangioblastomas, or in approximately $6 \%$ of all patients with spinal hemangioblastomas. These numbers do not differ significantly for patients with VHL-associated versus sporadic hemangioblastomas, because spinal nerve tumors account for $5 \%$ of VHL-associated hemangioblastomas and for $8 \%$ of patients with sporadic spinal hemangioblastomas.

\section{Clinical and Radiographic Features}

The clinical data of the six patients with spinal nerve hemangioblastomas are summarized in Table 1. All tumors were hypointense on T1-weighted images and exhibited moderate (Patient 1) to strong (Patients 2-6) contrast enhancement, which was in all cases inhomogeneous. On T2-weighted images, all lesions appeared inhomogeneous and hyperintense. In all lesions, tubular structures could be identified, and all but one tumor (Patient 4) caused foraminal widening. Angiography was performed in Patients 1 and 6 and revealed highly vascularized tumors supplied by extradural circulation. All tumors appeared as dumbbell-shaped lesions (Figs. 1-3). Only in those patients with known VHL was the definitive pathological diagnosis already suspected by the radiologist because of knowledge of the patient's history. The other patients (Patients 2 and 5) underwent surgery under an incorrect suspected diagnosis. In the case of Patient 5, the radiological interpretation of a metastasis led to an incomplete resection and a tumor recurrence after 3 years. In another patient (Patient 2), a neuroma was suspected primarily, which led the surgeon to incise the tumor capsule, with consequent profuse bleeding. Therefore, the surgeon had to leave behind a part of the tumor, which led to a symptomatic local recurrence after 2 years.

All of the tumors were operated on via a dorsal approach. The facet joints could be preserved only in two of the six patients (Patients 1 and 6), and in one patient (Patient 4), a partial facetectomy was performed. All tumors grossly were 


\begin{tabular}{|c|c|c|c|c|c|c|}
\hline $\begin{array}{l}\text { Patient } \\
\text { no. }\end{array}$ & $\begin{array}{l}\text { Age }(y r) / \\
\text { sex }\end{array}$ & $\begin{array}{l}\text { Spinal } \\
\text { nerve }\end{array}$ & VHL status & Preoperative symptoms & $\begin{array}{l}\text { Early postoperative } \\
\text { results }\end{array}$ & Late postoperative results \\
\hline 1 & $35 / M$ & $\mathrm{~T} 10$ & $\begin{array}{l}\text { VHL germline deletion } \\
2 \mathrm{~kb}\end{array}$ & $\begin{array}{l}\text { Spinal ataxia, back } \\
\text { pain, sensory deficit }\end{array}$ & $\begin{array}{l}\text { Paraplegia and } \\
\text { urinary incontinence } \\
\text { caused by hematoma }\end{array}$ & $\begin{array}{l}\text { After hematoma evacuation, } \\
\text { complete recovery }\end{array}$ \\
\hline 2 & $52 / \mathrm{M}$ & $\mathrm{C} 7$ & Negative & $\begin{array}{l}\mathrm{C} 7 \text { paresis and } \\
\text { paresthesia }\end{array}$ & $\begin{array}{l}\text { Same C7 paresis and } \\
\text { paresthesia }\end{array}$ & $\begin{array}{l}\text { Complete recovery, } \\
\text { symptomatic recurrence } \\
\text { after } 2 \mathrm{yr}\end{array}$ \\
\hline 3 & $40 / F$ & T6 & Mutation c452 G/T & Asymptomatic & Asymptomatic & Asymptomatic \\
\hline 4 & $48 / F$ & L5 & $\begin{array}{l}\text { Clinically positive, but } \\
\text { no mutation found }\end{array}$ & Radicular pain & Radicular pain & $\begin{array}{l}\text { Complete recovery to } \\
\text { normal }\end{array}$ \\
\hline 5 & $44 / \mathrm{M}$ & T6 & Negative & $\begin{array}{l}\text { Paraparesis, urinary } \\
\text { incontinence, radicular } \\
\text { pain }\end{array}$ & Complete recovery & $\begin{array}{l}\text { Symptomatic recurrence } \\
\text { after } 3 \mathrm{yr}\end{array}$ \\
\hline 6 & $46 / F$ & S1 & Mutation c505 T/C & Radicular pain & Low-grade S1 paresis & $\begin{array}{l}\text { Complete recovery, } \\
\text { symptomatic recurrence } \\
\text { after } 4 \mathrm{yr}\end{array}$ \\
\hline
\end{tabular}

${ }^{a}$ VHL, von Hippel-Lindau disease; kb, kilobases.

more yellowish than hemangioblastomas of the CNS, which usually appear macroscopically as bright orange-red.

During surgery, it was noted that the pathological anatomy of the tumors was always similar. In the cervical tumors, the vascular supply was from multiple feeders from the vertebral artery. In the other areas, the tumors had multiple feeding vessels from the surrounding muscle and bone tissue. A vascular supply from the spinal cord, as would be expected for a medullary lesion growing extradurally, was not observed. The tumors arose from a dorsal fascicle of the spinal nerve and pushed against the nerve ventrally. The tumors that were known to be hemangioblastomas beforehand could be removed en bloc in the following fashion. They were dissected stepwise from the surrounding tissue by coagulating and cutting all feeding vessels until the tumors lost turgor. Then they were dissected from the spinal nerve and removed. If the fascicle of origin could be identified, it also was removed. In no case did we remove the entire spinal nerve.

In most of the patients, moderate to severe blood loss was encountered during surgery. In the case of Patient 1, who had a particularly large tumor, significant intraoperative blood loss from feeders was unavoidable, although the tumor itself was not entered. Early postoperative hematoma occurred, despite a complete hemostasis before closure, which led to transient paraplegia and incontinence. Surgical evacuation of the hematoma resulted in complete recovery.

\section{Microscopic Features}

All tumors had the typical histomorphological features of hemangioblastoma. There was a dense network of capillaries that was surrounded by reticulin fibers (Fig. 4). Between these vessels were located interstitial cells that showed the characteristic foamy appearance. Immunohistochemical staining revealed expression of S100 protein, neuron-specific enolase, and neural cell adhesion molecule in a large portion of these cells. The histological and immunohistochemical findings therefore showed the regular features of hemangioblastoma.

\section{DISCUSSION}

Hemangioblastomas occur mainly in the posterior fossa and in the spinal cord. Most spinal hemangioblastomas arise in the dorsal root entry zone and grow mainly in an intramedullary fashion; however, a portion of the tumors may extend into extramedullary tissue, such as nerve roots or the filum terminale, which has been described extensively by many authors in major series $(5,7,9,13-16,19-22,25,26)$.

Extradural hemangioblastoma of the spinal nerve is a tumor entity that so far has not been well described, with only eight case reports published so far $(4-6,11,16,20)$, some of which were partly intradural and thus not definitely of spinal nerve origin $(16,20)$. Here, we present a series of six patients with extradural hemangioblastomas of a spinal nerve. This relatively high number could be explained by the fact that our hospital serves as a referral center for patients with VHL and hemangioblastomas. Altogether, extradural spinal nerve hemangioblastomas can be considered to be rare tumors. Our study reveals that hemangioblastoma of the spinal nerve comprises $2 \%$ of all CNS hemangioblastomas and $6 \%$ of all spinal hemangioblastomas. However, these calculated proportions 

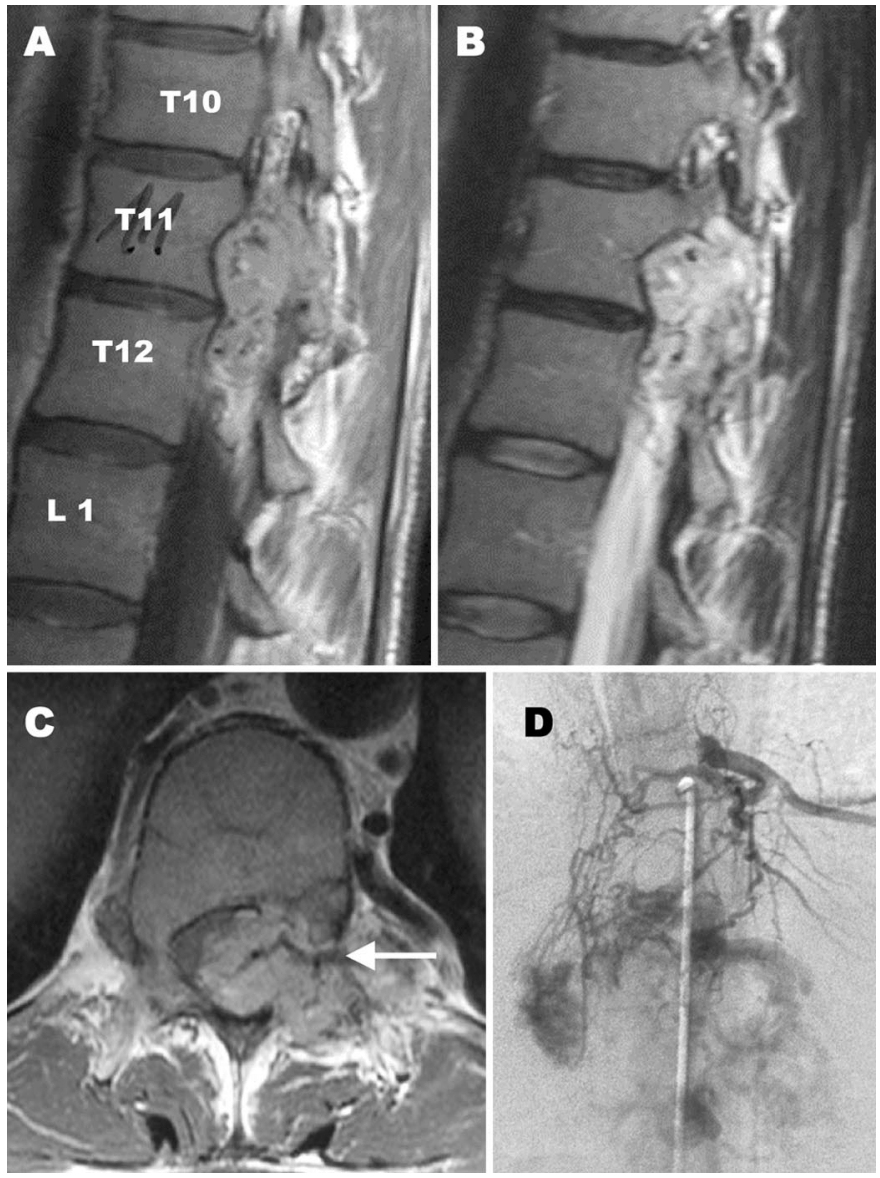

FIGURE 1. Patient 1, a 35-year-old man with a known history of VHL. Because the lesion was at the level of the left 11th thoracic neuroforamen, it was classified primarily as a hemangioblastoma. A, sagittal contrastenhanced T1-weighted MRI scan of the spine shows an inhomogeneous enhancing lesion. The neuroforamen and the vertebral body are scalloped. $\mathrm{B}$, sagittal T2-weighted MRI scan shows inhomogeneous tumor matrix tubular structures, which are visible within the tumor. C, axial contrastenhanced T1-weighted MRI scan reveals the tumor compressing the spinal cord on one side and growing outside the spinal canal into the extradural space on the other side. The tubular structures are even easier to recognize, and a large vessel runs through the tumor (arrow). D, digital subtraction angiogram exhibits a highly vascular lesion supplied from a dilated radiculospinal artery arising from the left $T 10$ radicular artery.

are approximations, because although we also operate on asymptomatic tumors that show progression in serial MRI scans, we cannot exclude the possibility that we missed some asymptomatic nonprogressive extradural hemangioblastomas in our calculation. Thus, the real proportion of spinal nerve hemangioblastomas may be even higher, because only surgical patients were included in the study.

\section{Radiological Features and Diagnostic Pitfalls}

Spinal nerve hemangioblastomas to date are not specifically described in the neuroradiological literature. As our study
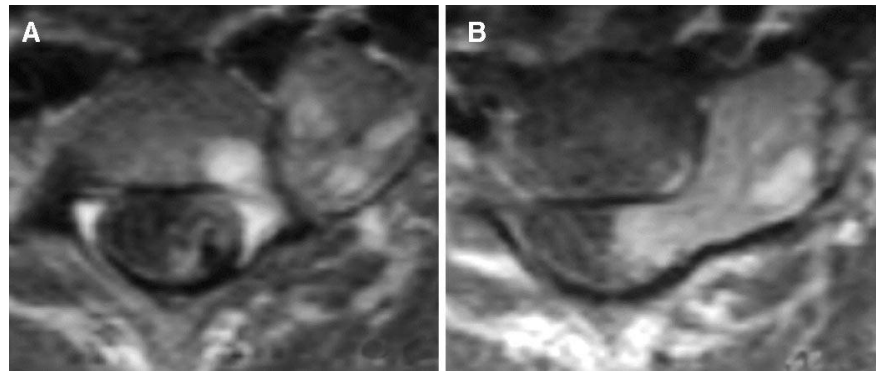

FIGURE 2. Patient 3, a 40-year-old woman with a known history of VHL. The tumor was classified primarily as a hemangioblastoma. A, T1-weighted MRI scan of the spine shows a dumbbell-shaped, inhomogeneous, mainly hypointense lesion at the level of the 6th cervical neuroforamen, with a major component extending through the neuroforamen into the paravertebral space. The lesion pushes the spinal cord to the contralateral side and seems to be arising extradurally. The neuroforamen is scalloped, and the vertebral artery is dislocated ventrally. B, after application of gadolinium, the tumor exhibits inhomogeneous contrast enhancement.

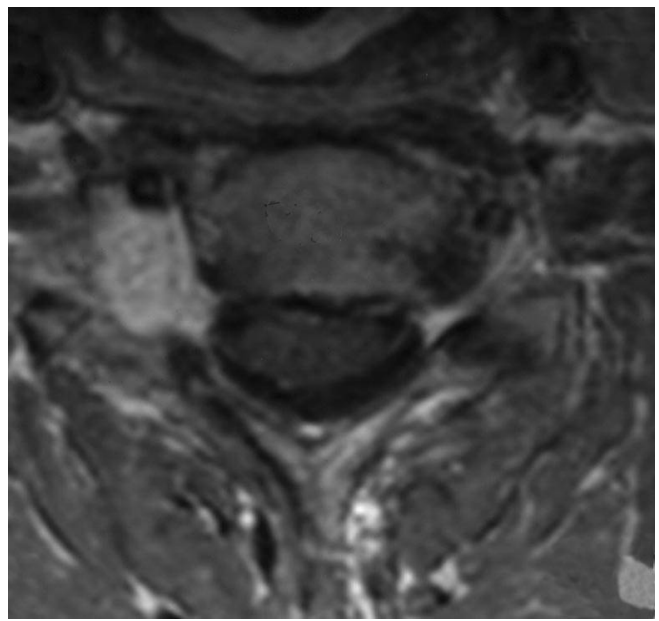

FIGURE 3. Patient 2, a 52-year-old man who had no stigmata of VHL but had a right triceps paresis paresthesia in the right $\mathrm{C} 7$ dermatoma. The T1-weighted MRI with contrast shows a strongly enhancing tumor in the right C7 neuroforamen pushing the vertebral artery ventrally. The contrast enhancement is somewhat inhomogeneous, and tubular structures can be seen. The tumor was classified primarily as a neuroma. During surgery, the tumor was entered, which led to major bleeding problems, and consequently, only a partial resection of the tumor was possible. The patient recovered completely from his symptoms. Histopathological examination revealed a World Health Organization Grade 1 hemangioblastoma. After 2 years, the patient experienced the same symptoms, and an MRI scan revealed a recidive tumor. This time, the tumor was removed completely, and the patient has been without recidive disease for 5 years.

shows, these tumors are certainly a diagnostic challenge and easily can be mistaken for metastases or neuromas, which can lead to severe surgical complications. However, after reviewing these six cases, some specific radiographic features of spinal nerve hemangioblastomas can be identified retrospectively. 


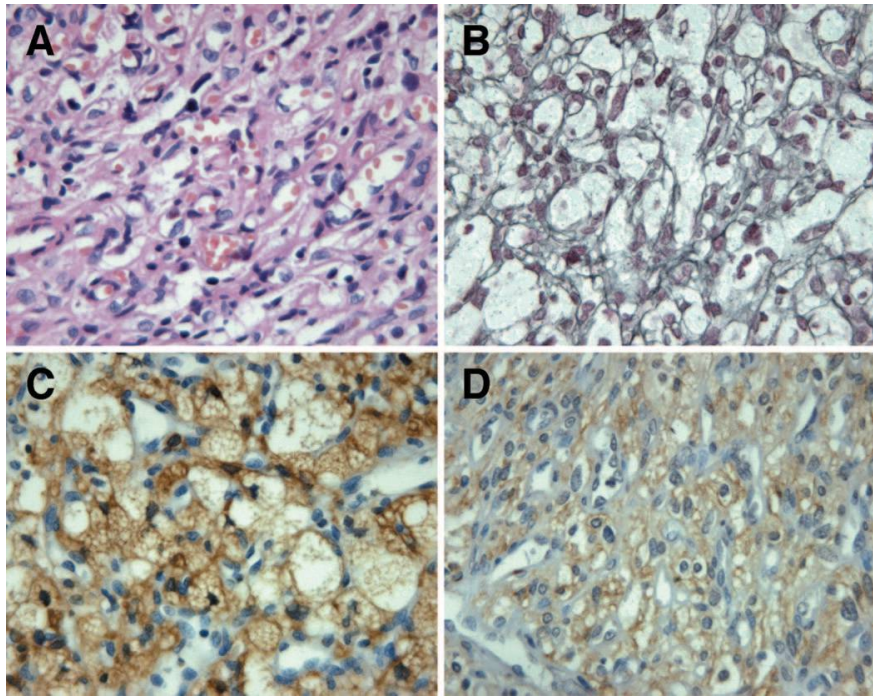

FIGURE 4. Photomicrographs demonstrating histopathological features of hemangioblastomas. A, routine hematoxylin and eosin stain showing numerous capillaries and stromal cells that reveal the typical foamy appearance. B, reticulin fiber stain revealing the dense fiber network surrounding the capillaries. $\mathrm{C}$ and $\mathrm{D}$, immunohistochemistry revealing the expression of protein S100 (C) and neural cell adhesion molecule (D) in the stromal cells (original magnification, $\times 40$ ).

Spinal nerve hemangioblastomas appear as dumbbellshaped, contrast-enhancing lesions. In contrast to hemangioblastomas of the brain, which often appear with a vascular nodule and concomitant cyst, as also is seen commonly in spinal cord lesions, the extradural hemangioblastomas were solid. The inhomogeneous appearance on T2-weighted images, which also has been observed by other authors (6), is a result of prominent foci of tubular structures with flow void resulting from the highly vascular mass. The intensive contrast enhancement also is a result of prominent tumor vessels with dilated feeding arteries and draining veins. In contrast, schwannomas usually enhance intensely and fairly homogeneously, although some of them enhance peripherally, sparing the central part of the lesion. Schwannoma and extradural hemangioblastoma typically can scallop the vertebral bodies with widening of the neural foramina, as was seen in five of our six patients. In contrast, metastasis or malignant nerve sheath tumors may infiltrate and erode the bony structures. The differential diagnosis between hemangioblastoma and plexiform neurofibroma can be difficult, because both entities envelop the dorsal sensory root and show typically irregular contrast enhancement and inhomogeneous T2-weighted appearance, but the flow voids seems to be more frequent in hemangioblastoma. It is important to know whether the patients have VHL, because in these patients, neuromas usually do not occur, and the diagnosis of hemangioblastoma thus can be established on a clinical basis. In those spinal nerve tumors in which the images are suggestive of a sporadic hemangioblastoma because of tubular structures, angiography should be considered and embolization may be possible.

\section{Surgical Features and Pitfalls}

Generally, removal of a spinal hemangioblastoma is associated with a good outcome. Only in two of our patients did a temporary neurological deterioration occur. None of our patients had disabling long-term motor deficits, despite the fact that in most of them, those nerve fascicles that were involved in the tumor were removed. The explanation for this phenomenon may be that generally only dorsal root fascicles were involved in the tumor.

In all patients, there was more blood loss than one would expect for a neuroma (except for Patient 6, in whom preoperative embolization was performed), in contrast to our experience with intradural hemangioblastomas, where we have observed no major bleeding problems in a series of 28 consecutive patients (23). In two patients (Patients 1 and 2), the blood loss led to clinically significant coagulopathy, making the surgery more difficult in Patient 2 and leading to postoperative bleeding with transient paraparesis in Patient 1. Avoiding excessive blood loss by not entering the tumor capsule as long as the feeding vessels are coagulated is the key to successful surgery and low morbidity when operating on these vascular tumors.

Unfortunately, in three of the six patients (Patients 2, 5, and 6), we observed local tumor recurrence, in contrast to our experience with intradural hemangioblastomas, in which we have observed no recurrences in a series of 28 consecutive patients (23). The reason for this was that in two patients (Patients 2 and 5), the initial radiographic assessment of the lesion was incorrect. The suspicion of a schwannoma led the surgeon to incise the tumor capsule in Patient 2. This was followed by profuse bleeding, which finally resulted in the surgeon having to leave behind a part of the tumor. It is likely that a different surgical strategy would have been chosen if the diagnosis of hemangioblastoma had been established initially; the surgeon would not have incised the tumor capsule but rather would have dissected around the tumor capsule by coagulating all feeding vessels, as is recommended for hemangioblastoma (23). The radiographic diagnosis of a metastasis in Patient 5 also led the surgeon to leave behind a part of the tumor. In Patient 6, local tumor recurrence occurred, despite apparently complete resection of the primary tumor. In general, it is important always to try to identify the fascicle of origin and to resect it, because simply dissecting the tumor from the nerve can result in recurrence.

\section{Pathological Features}

Histologically, the tumors described here showed the characteristic morphological and immunohistochemical staining features of hemangioblastomas observed in the CNS. The origin of the interstitial cells that are the neoplastic cells proper in hemangioblastoma has not yet been resolved. The localization of these tumors virtually exclusively in the CNS and, as we demonstrate here, only rarely in the nearby CNS, as well as the expression of a number of neural antigens, such as neuronspecific enolase, neural cell adhesion molecule, and S100 pro- 
tein, may suggest that these cells derive from the neural tube or neural crest; however, to our knowledge, there are no reports that support this hypothesis. Recently, Vortmeyer et al. (24) reported that hemangioblastomas in VHL patients consist of developmentally arrested hemangioblasts that have the potential to differentiate into primitive vascular structures as well as into red blood cells. Although this report sheds some light on the origin of these tumors, the reason for the localization of these tumors predominantly in the CNS remains to be elucidated. It could be speculated that the central nervous environment may favor the development of hemangioblastoma in predisposed individuals. Further studies are needed to clarify the nature and origin of the interstitial cells.

\section{CONCLUSIONS}

Hemangioblastomas of the spinal nerve harbor diagnostic and surgical pitfalls. Therefore, they should be considered as their own clinical entity. In general, the tumors are surgically challenging, and clinically significant bleeding as well as local tumor recurrence is more common than in intradural hemangioblastomas. We suggest that because of the surgical consequences, hemangioblastoma always be considered to be an important radiological differential diagnosis for nerve sheath tumors, especially in cases with vascular flow void and inhomogeneous contrast uptake. When this vascular tumor is suspected, angiography can bring clarification. Preoperative embolization to avoid significant blood loss, as occurred in two of our six patients, should be considered. Spinal nerve hemangioblastomas usually can be removed completely without major surgical morbidity, as long as the surgeon respects the plane between the tumor capsule and the surrounding tissue.

\section{REFERENCES}

1. Bohling T, Plate KH, Haltia M, Alitalo K, Neumann HP: Von Hippel-Lindau disease and capillary hemangioblastoma, in Kleihues P, Cavenee WK (eds): Pathology and Genetics of Tumours of the Central Nervous System. Lyon, World Health Organization, 2000, pp 223-226.

2. Brodkey JA, Buchignani JA, O'Brien TF: Hemangioblastoma of the radial nerve: Case report. Neurosurgery 36:198-201, 1995.

3. Browne TR, Adams RD, Roberson GH: Hemangioblastoma of the spinal cord: Review and report of five cases. Arch Neurol 33:435-441, 1976.

4. Chazono M, Shiba R, Funasaki H, Soshi S, Hattori A, Fujii K: Hemangioblastoma of the L-5 nerve root: Case illustration. J Neurosurg Spine 90:160, 1999.

5. Chu BC, Terae S, Hida K, Furukawa M, Abe S, Miyasaka K: MR findings in spinal hemangioblastoma: Correlation with symptoms and with angiographic and surgical findings. AJNR Am J Neuroradiol 22:206-217, 2001.

6. Escott E, Kleinschmidt-DeMasters B, Brega K, Lillehei K: Proximal nerve root spinal hemangioblastomas: Presentation of three cases, MR appearance and literature review. Surg Neurol 61:262-273, 2004.

7. Farneti M, Ferracini R, Migliore A, Trapella G, Veronesi V: Isolated hemangioblastoma of the filum terminale: Case report. J Neurosurg Sci 45:58-62, 2001.

8. Freude S, Hausmann J, Hofer M, Pham-Mitchell N, Campbell IL, Staeheli P, Pagenstecher A: Borna disease virus accelerates inflammation and disease associated with transgenic expression of interleukin-12 in the central nervous system. J Virol 76:12223-12232, 2002.
9. Giannini C, Scheithauer BW, Hellbusch LC, Rasmussen AG, Fox MW, McCormick SR, Davis DH: Peripheral nerve hemangioblastoma. Mod Pathol 11:999-1004, 1998.

10. Gläsker S, Bender BU, Apel TW, Natt E, van Velthoven V, Scheremet R, Zentner J, Neumann HP: The impact of molecular genetic analysis of the VHL gene in patients with hemangioblastomas of the central nervous system. J Neurol Neurosurg Psychiatry 67:758-762, 1999.

11. Hermier M, Cotton F, Saint-Pierre G, Jouvet A, Ongolo-Zogo P, Fischer G, Froment JC: Myelopathy and sciatica induced by an extradural S1 root hemangioblastoma. Neuroradiology 44:494-498, 2002.

12. Ho VB, Smirniotopoulos JG, Murphy FM, Rushing EJ: Radiologic-pathologic correlation: Hemangioblastoma. AJNR Am J Neuroradiol 13:1343-1352, 1992.

13. Hurth M: Intraspinal hemangioblastomas [in French]. Neurochirurgie 21[Suppl 1]:1-136, 1975.

14. Ismail SM, Cole G: Von Hippel-Lindau syndrome with microscopic hemangioblastomas of the spinal nerve roots: Case report. J Neurosurg 60:12791281, 1984.

15. Lonser R, Wait S, Butman J, Vortmeyer A, Walther M, Governale L, Oldfield E: Surgical management of lumbosacral nerve root hemangioblastomas in von Hippel-Lindau syndrome. J Neurosurg 99:64-69, 2003.

16. McEvoy AW, Benjamin E, Powell MP: Hemangioblastoma of a cervical sensory nerve root in von Hippel-Lindau syndrome. Eur Spine J 9:434-436, 2000 .

17. Neumann HP, Eggert HR, Scheremet R, Schumacher M, Mohadjer M, Wakhloo AK, Volk B, Hettmannsperger U, Riegler P, Schollmeyer P: Central nervous system lesions in von Hippel-Lindau syndrome. J Neurol Neurosurg Psychiatry 55:898-901, 1992.

18. Neumann HP, Eggert HR, Weigel K, Friedburg H, Wiestler OD, Schollmeyer $\mathrm{P}$ : Hemangioblastomas of the central nervous system: A 10-year study with special reference to von Hippel-Lindau syndrome. J Neurosurg 70:24-30, 1989.

19. Raghavan R, Krumerman J, Rushing EJ, White CL III, Chason DP, Watson ML, Coimbra C: Recurrent (nonfamilial) hemangioblastomas involving spinal nerve roots: Case report. Neurosurgery 47:1443-1448, 2000.

20. Rohde V, Voigt K, Grote EH: Intra-extradural hemangioblastoma of the cauda equina. Zentralbl Neurochir 56:78-82, 1995.

21. Seki T, Hida K, Iwasaki Y: Hemangioblastoma of the spinal nerve root [in Japanese]. No Shinkei Geka 29:331-334, 2001.

22. Tibbs RE Jr, Harkey HL, Raila FA: Hemangioblastoma of the filum terminale: Case report. Neurosurgery 44:221-223, 1999.

23. Van Velthoven V, Reinacher PC, Klisch J, Neumann HP, Gläsker S: Treatment of intramedullary hemangioblastomas, with special attention to von Hippel-Lindau disease. Neurosurgery 53:1306-1314, 2003.

24. Vortmeyer AO, Frank S, Jeong SY, Yuan K, Ikejiri B, Lee YS, Bhowmick D, Lonser RR, Smith R, Rodgers G, Oldfield EH, Zhuang Z: Developmental arrest of angioblastic lineage initiates tumorigenesis in von Hippel-Lindau disease. Cancer Res 63:7051-7055, 2003.

25. Wisoff HS, Suzuki Y, Llena JF, Fine DI: Extramedullary hemangioblastoma of the spinal cord: Case report. J Neurosurg 48:461-464, 1978.

26. Wolbers JG, Ponssen H, Kamphorst W: Hemangioblastoma of the cauda equina. Clin Neurol Neurosurg 87:55-59, 1985. 